\title{
Enhancing the Physical Modeling Capability of Open-Source MFIX-DEM Software for Handling Particle Size Polydispersity: Implementation and Validation
}

\author{
Shaohua Chen ${ }^{\mathrm{a}, *}$, Manogna $\mathrm{Adepu}^{\mathrm{a}, *}$, Heather Emady ${ }^{\mathrm{a}}$, Yang Jiao ${ }^{\mathrm{a}, * *}$, Aytekin \\ $\mathrm{Gel}^{\mathrm{b}}$ \\ ${ }^{a}$ School for Engineering of Matter, Transport and Energy, Arizona State University, \\ Tempe, AZ 85287 \\ ${ }^{b}$ School of Computing, Informatics, Decision Systems Engineering, Arizona State \\ University, Tempe, AZ 85287
}

\begin{abstract}
Multiphase flows are ubiquitous in many industrial processes. The inherent coupling of different phases poses many unique challenges in predicting and effectively controlling these processes. Hence, computational modeling and simulation offers a viable approach to overcome these challenges. In this study, we present recent development efforts for enhancing the physical modeling capabilities of an open-source computational modeling tool for real life industrial multiphase processes by enabling particle-size polydispersity and demonstrating with an associated validation study. The proposed implementation was performed in MFIX open-source framework due to its unique feature of tightly integrated computational fluid dynamics and discrete element method solvers for simulating coupled continuum fluid and granular flows. We have implemented the polydispersity feature in a minimally invasive way and provided means to allow easy specification of an arbitrary particle size distribution function, which also enables the user to easily handle an arbitrary number of solid phases, each possessing a distinct arbitrary particle-size distribution. To establish the credibility
\end{abstract}

\footnotetext{
*Authors contribute equally to this work.

** Corresponding author

Email address: yang.jiao.2@asu.edu (Yang Jiao)
}

Preprint submitted to Powder Technology

April 12, 2017

(C) 2016. This manuscript version is made available under the Elsevier user license http://www.elsevier.com/open-access/userlicense/1.0/ 
of improvements, we have carried out a preliminary verification and validation $(\mathrm{V} \& \mathrm{~V})$ study for the polydispersity feature by employing a hopper bin discharge problem, which is frequently encountered in industrial applications. Specifically, two types of micro glass beads with distinct size distributions are used to fill the hopper in two possible packing arrangements, i.e., well-mixed and layered configurations, with varying mass (particle number) ratios. The experimentally obtained discharge dynamics (e.g., normalized discharge mass fraction for one of the phases versus the overall discharge mass fraction) for different systems is found to be in excellent agreement with the corresponding simulation results.

Keywords: Granular flow, polydisperse particles, discharge hopper, open-source modeling and simulation, discrete element method (DEM), MFIX-DEM

\section{Introduction}

2 Multiphase flows are ubiquitous in many industrial processes. Common examples include wet granulation, coating, spray drying, pneumatic conveying,

4 and fluidization. The inherent coupling of these different phases poses many unique problems in predicting, and effectively controlling these processes. Com-

6 putational simulations are often employed for this purpose. Typically, separate scientific computational modeling software for discrete element method (DEM) 8 to solve the solid phase, such as LIGGGHTS [1, 2, 3, 4, and computational fluid dynamics (CFD) to solve the continuum fluid phase, such as OpenFOAM 10 [5, 6, 7], are externally coupled to model the governing physics in such flows.

Here, we present our recent developments for enhancing MFIX [8, 9, 10], 12 a suite of open-source software for multiphase flow simulations including both the two-fluid model (TFM) and the coupled CFD-DEM model, developed and

14 maintained by the National Energy Technology Laboratory (NETL) of the U.S. Department of Energy. For the TFM model, both the carrier fluid and the solid particle phases are treated as a continuum, and the Eulerian-Eulerian framework solver is utilized to solve the associated conservation equations for both phases. 
18 For the coupled CFD-DEM model, MFIX is a unique tool that provides a unified framework for a single user interface and code base to simulate a diverse range of multiphase flow problems [11, 12]. Hence, MFIX eliminates the need to externally couple two separate modeling software. Instead, MFIX utilizes the

22 TFM solver (i.e., Two-Fluid Model-based Eulerian-Eulerian framework solver) for performing the computations of the carrier fluid and the DEM solver (which

24 is the Lagrangian framework based solution process) for handling particles.

Since its initial release, MFIX has been successfully employed to model a variety of multiphase flow problems relevant to industrial and energy-related applications [13, 14, 15, 16, 17, 18, 19, 20, 21. Due to the inherent complexity and diverse range of spatial/temporal scales involved in multiphase flow simulations, several initiatives were carried out to reduce the time-to-solution through 30 performance improvements [22, 23, 24, 25, 26, 27, 28, 29, 30,. Also, a number of studies have been carried out to systematically perform uncertainty quantification analysis in multiphase flow simulations with MFIX [31, 32, 33, 34] to improve the credibility of the prediction capability.

Despite these enhancements and improvements, the lack of computationally efficient methods to simulate polydisperse systems (heterogeneity of sizes) has

36 posed some problems when modeling industrial applications. For example, even with the recent 2016-1 release version of MFIX-DEM, each distinct solid phase

38 is only allowed to possess a unique particle size, making it extremely difficult to simulate a true polydisperse system, in which each particle possesses a unique

40 size (e.g., particle diameter). Accurately capturing the particle size distribution is crucial to processes involving denser granular flows and jammed granular packings [35, 36]. More importantly, the capability to handle polydisperse systems easily is often available in other open-source and commercial DEM software

44 (e.g., LIGGGHTS [37] and EDEM [38]), and also in other particle-based simulation techniques, such as event-driven molecular dynamics [39, 40] and Monte 46 Carlo simulations 41.

In the current paper, we present our recent work on enhancing MFIX-DEM's 48 physical modeling capability for handling particle-size polydispersity for the 
pure DEM mode. Specifically, we have modified the data structure and created new subroutines to separately handle geometrical and physical parameters of the solid phase particles. This approach has enabled MFIX-DEM to easily handle an arbitrary number of solid (particle) phases where the particles in each phase are allowed to possess a distinct arbitrary particle-size distribution. Several new 54 input keywords have been introduced to allow easy specification of the particlesize distribution function for each phase. The user can select either one of the built-in distributions (e.g., normal, log-normal, and uniform) or user-defined distributions (that can be specified via the restart files). To establish the credibility of the new improvements implemented, a preliminary verification and validation $(\mathrm{V} \& \mathrm{~V})$ study is performed using hopper bin discharge experiments, which is a commonly used configuration with many industrially relevant applications. In particular, we use two types of micro glass beads with distinct size distributions 62 to fill the hopper in two possible packing arrangements, i.e., well-mixed and layered configurations, with varying mass (particle number) ratios. The experi64 mentally obtained discharge dynamics (e.g., normalized discharge mass fraction for one of the phases vs. the overall discharge mass fraction) for different systems is found to be in excellent agreement with the corresponding simulation results, which clearly validates the newly implemented polydispersity features.

68 The new features for handling polydispersity will be available in a future version of MFIX.

70

The rest of the paper is organized as follows. In Sec. 2, a brief overview of the implementation for polydispersity is presented, followed by a verification study using a discharge hopper. In Sec. 3, we present the details of our hopper bin discharge experiments using micro glass beads, which were designed for the validation study as discussed in the following section. In Sec. 4, we systematically validate the newly implemented polydispersity feature using the experimental 76 data. Both our hopper discharge experimental results and MFIX-DEM simulation results are reported and are observed to be in excellent agreement. In Sec. 78 5, we provide concluding remarks. 


\section{Implementation}

80

82

88 forces.

Figure 1: Schematic illustration of MFIX-DEM's unified framework coupling both the CFD solver and DEM solver under a single user interface with the major subroutines being called. The subroutines modified for implementing the polydispersity feature are highlighted in the red boxes. Adapted from Fig. 1 in Ref. [27.

Before discussing our implementation for polydispersity, a brief background on MFIX-DEM is provided in this section. As mentioned in Sec. 1, MFIX (https://mfix.netl.doe.gov) is a unique open-source computational fluid dynamics (CFD) software for modeling multiphase flows, developed and maintained by the U.S. Department of Energy over several decades. It offers a unified framework for CFD and discrete element method (DEM) and is extensively used in diverse areas, from coal gasification to volcano explosion modeling [13, 14, 25, 42, 43, 44, 45, 46, 47.

Fig. 11illustrates the overall workflow of MFIX when the CFD and DEM solution processes are invoked for solving granular flows coupled with a continuum fluid [48, 49]. As seen from the flowchart in Fig. 1] once the coupling is enabled, at a particular CFD time step, the conservation equations are solved iteratively by the CFD solver, and the resulting drag forces and fluid-solid momentum transfer are subsequently computed and passed to the DEM solvers. Within a single CFD time step, multiple DEM time steps are carried out to integrate the equations of motion associated with the particles for the given contact and drag

\subsection{Modification of data structure and subroutines}

Our implementation for polydispersity aimed to minimize the invasive structural changes in the algorithm and the original MFIX-DEM code. In the latest release of MFIX-DEM (i.e., 2016-1), each distinct solid phase is only allowed to possess a unique particle size, which is saved in the array D_PO, whose size is the

maximum number of solid phases in the system. Each solid particle is assigned a 
unique phase index based on its diameter, which helps in phase-specific physical properties of the particles (e.g., material density, Young's modulus, coefficient of friction, coefficient of restitution, etc.) to be retrieved and employed in the subsequent computation of particle contact forces.

The basic idea of our new implementation is to separately save the particle geometrical parameters (i.e., diameters), which are particle-specific and the phase-specific physical parameters. In particular, we modified the array D_Po such that it saves the diameter of each particle in the system, thus possessing a size of the total particle number $N$. For example, D_PO(i) now gives the diameter of particle $i$, instead of the diameter of particles for phase $i$. Accordingly, the phase index of each particle is not assigned according to its diameter, but assigned based on its physical properties, e.g., material density. The change of data structure for saving and retrieving an individual particle diameter (or radius) affects a number of subroutines which utilize D_PO(i) as input for subsequent calculations. As summarized in Fig. 1, the modified subroutines include those for particle force/torque calculation (calc_force_dem.f), solid-gas momentum transfer calculation (calc_drag_des.f), grid cell locator (desgrid_mod.f) and neighbor locator (neighbour.f). We note the modifications do not affect any of the underlying governing equations for the system nor specific contact force models to be utilized.

The new implementation offers three types of built-in probability distribution functions (PDF) for size, including normal, log-normal and uniform distributions. However, the addition of new distributions can easily be done through the DES/RANDOMNO_mode.F subroutine. For a user-specified distribution, the particle diameter information is provided in the restart files (*.RES) and is read in during the initialization stage. In addition, users can specify the particle size distribution for both the initial condition (IC) and mass-in-flow (MIF) boundary conditions (BC). This allows the simulation of complex multiphase flow processes during which additional solids are injected into the system. This implementation is achieved by introducing two sets of new keywords, respectively, 34 for ICs and MIF BCs. The new polydispersity feature will be available in the 
next release version of MFIX with detailed documentation and tutorial cases.

\subsection{Verification of the polydispersity implementation}

As part of our preliminary $\mathrm{V} \& \mathrm{~V}$ effort, we first verified the new implementation by simulating particle discharge from a 3D hopper with equal-sized spherical beads. The 2016-1 release version of MFIX is also used to simulate the same system for comparison. The hopper contains a cylinder part of height $5 \mathrm{~cm}$ and diameter $6 \mathrm{~cm}$, connected to a cone part with height $5 \mathrm{~cm}$ and angle of 30 degrees. The bottom of the hopper cone is further connected to a short cylinder tube of height $0.2 \mathrm{~cm}$ and diameter $0.5 \mathrm{~cm}$. The monodisperse beads possess a diameter of $0.068 \mathrm{~cm}$, spring constant of $2.5 \times 10^{5} \mathrm{~g} / \mathrm{s}^{2}$, friction coefficient of 0.01 and coefficient of restitution of 0.5 .

The initial configuration for the discharge simulation is obtained by first filling the hopper with 15540 beads from the top (through the mass-in-flow boundary condition), while the bottom is kept closed (through the no-flow boundary condition). The resulting packing is allowed to settle completely, to dissipate the kinetic energy. The stable packing at the end of settling is used as the initial configuration for the discharge simulation until the hopper is fully discharged.

Table 1: Verification simulation details for the discharge hopper with monodisperse spherical beads.

Figure 2: Discharge dynamics for a 3D hopper with equal-sized spherical beads. The discharged mass vs. discharge time curves obtained from both the 2016-1 MFIX and our new implementation agree well with one another.

152 Tab. 1 summarizes the simulation details, including the number of particles, domain decomposition configuration and total CPU hours required for full discharge. Fig. 2 shows the discharged mass vs. time for the monodisperse system, obtained using the 2016-1 MFIX-DEM release and our new polydisperse implementation derived from the 2016-1 release. It can be seen that in both cases, the initial discharge rates are relatively small and stabilize $\sim 2$ seconds after 
discharge starts. The discharged mass fractions for the two cases slightly deviate from one another due to uncertainty in the initial packing configurations. The overall discharge dynamics agree very well with one another. Also, the total computational cost in terms of wall-clock CPU hours for the simulations are also comparable in the two cases. Hence, these results verify the correctness of our new implementation.

\section{Hopper Discharge Experiment}

In the next step of our $\mathrm{V} \& \mathrm{~V}$ effort, to validate our polydispersity implementation, hopper discharge experiments were carried out using two types of polydisperse particles with varying initial packing configurations. Detailed discharge dynamics were obtained for both types of particles for comparison with simulation results. The hopper used in the experiments (shown in Fig. 30 is 3D printed using a Stratasys Dimension 1200es SST (Stratasys Ltd. MN, USA), with ABSplus thermoplastic. It contains a cylinder of $12.5 \mathrm{~cm}$ height and 12.5 $\mathrm{cm}$ diameter, connected to a cone with a height of $3.5 \mathrm{~cm}$ and $55^{\circ}$ cone angle. The bottom of the hopper cone is further connected to a short cylinder of height

${ }_{174} 1.3 \mathrm{~cm}$ and diameter $2.5 \mathrm{~cm}$. The hopper is leveled and clamped to a support stand. A glass slide gate is used to close and open the hopper outlet, to fill and discharge, respectively.

Figure 3: Experimental setup of hopper: Clamped 3D printed hopper with a beaker, for collecting discharged particles, placed on an analytical balance to measure the mass of discharged particles for sampling.

The granular materials used in the experiments are silica beads (soda-lime silica glass). The silica beads are purchased from Potters Industries, PA, USA; with particle density $2.5 \mathrm{~g} / \mathrm{cm}^{3}$ in different sizes. The particles are further sieved to narrow the particle size distribution (PSD). The sieved particles are analyzed using a Malvern Morphologi G3SE (Malvern Instruments Ltd, UK), and two distinct sized polydisperse silica beads with a bi-modal size distribution 
Figure 4: A bi-modal particle size distribution (i.e., number frequency vs. particle sizes) was employed, which includes two normal distributions for fine and coarse silica bead particles.

(shown in Fig. 4) are used for the experiments. Specifically, the system can be

184

considered to contain two phases of solid particles, each possessing a distinct normal size distribution, with the same physical properties. The fine particles possess a normal distribution with a mean of $1.5 \mathrm{~mm}$ and standard deviation of $0.3 \mathrm{~mm}$, and the coarse particles possess a normal distribution with a mean of $2.9 \mathrm{~mm}$ and standard deviation of $0.1 \mathrm{~mm}$ (see Fig. 5 .

While the particles discharge through the hopper, they become tribocharged and tend to stick to the walls of the hopper. To remove any charging of the particles during discharge, the particles are treated with an anti-static solution. The anti-static solution is prepared by dissolving $1 \mathrm{ml}$ of ASA antistatic agent (Electrolube, UK) into $100 \mathrm{ml}$ of ethanol. No change in the discharge dynamics and segregation is observed before and after the particles are coated with the anti-static solution.

Figure 5: Silica beads: Normal distribution of the fine particles with mean of $1.5 \mathrm{~mm}$ and standard deviation of $0.3 \mathrm{~mm}$, colored in red; and the coarse particles with a mean of $2.9 \mathrm{~mm}$ and standard deviation of $0.1 \mathrm{~mm}$, colored in green.

The masses of the fine and coarse particles used in the experiments are $580 \mathrm{~g}$ and $420 \mathrm{~g}$, respectively. Two initial packing configurations are prepared using the fine and coarse particles. In the first configuration, the fine and coarse particles are well mixed. For this, both the fine and coarse particles are divided into 10 equal portions. One portion of fine particles is mixed with one portion of coarse particles using a Turbula T2F Shaker-Mixer (Glen Mills Inc., 202 NJ, USA). The same procedure is repeated with the remaining 9 portions to produce 10 equal portions of well-mixed fine and coarse particles. The hopper outlet is closed, and each portion is loaded slowly into the hopper, to minimize segregation due to free fall. The particle bed is leveled after each portion is loaded. In the second configuration, the coarse particles are first packed at the 
bottom of the hopper (with the outlet closed). Then the fine particles are loaded

on top of the coarse particles to form a layered packing. Each layer is leveled after loading into the hopper. We note that these configurations were also used by Ketterhagen et al., (2007) [50].

Figure 6: Particle arrangement: (a) First discharged sample from the well-mixed configuration. An approximately equal number of fine and coarse particles are discharged. (b) Last discharged sample from the well-mixed configuration. A majority of fine particles are discharged. (c) First discharged sample from the layered configuration. Only coarse particles are discharged. (d) Last discharged sample from the layered configuration. A mixture of both fine and coarse particles are discharged.

The discharge experiment is carried out using a discontinuous sampling method [51]. The outlet of the hopper is opened, and samples of equal mass are collected using the discontinuous sampling method. The initial and final arrangement of particles, for both mixed and layered configurations, are shown in Fig. 6. The collected samples are sieved and weighed to determine the mass of fine and coarse particles. Then the discharged mass fractions of both the fine and the coarse particles are determined, and the segregation data is plotted as the normalized mass fraction of fines $\left(\gamma_{f}^{N}\right)$ and the overall discharged fractional mass $(\bar{\gamma})$, using Equations 1 and 2 .

$$
\begin{gathered}
\gamma_{f}^{N}=\gamma_{f}^{\text {discharge }} / \gamma_{f}^{0} \\
\bar{\gamma}=m^{\text {discharge }} / m^{0}
\end{gathered}
$$

where, $\gamma_{f}^{N}$ is the normalized fine mass fraction of the discharged particles, $\gamma_{f}^{\text {discharge }}$ is the fine mass fraction of the discharged particles, $\gamma_{f}^{0}$ is the fine mass fraction in the initial packing configuration, $\bar{\gamma}$ is the overall discharged mass fraction, $m^{\text {discharge }}$ is the total discharged mass and $m^{0}$ is the mass of the initial particle packing. During the experiments with the well-mixed configuration, segregation occurs while loading. Therefore, each experiment is replicated five times to minimize the effects of any non-homogeneous regions on 
discharge segregation results, for both well mixed and layered configurations. The experimental results are reported in the subsequent section to validate our polydispersity implementation.

\section{Comparison of Experiment and Simulation Results}

In this section, we present the validation of our newly implemented polydispersity feature by replicating the experimental setup in Sec. 3. For the simulations, we consider that the glass beads possess model properties consistent with the previous studies [50]. As discussed in Sec. 2, in order to handle size distribution more efficiently in the simulation, a maximum value and a minimum value are specified for each size distribution. The fine-to-coarse particle mass ratio is $0.58: 0.42$, corresponding to a fine-to-coarse number ratio of $8: 1$. 238 The parameters for the particle phases used in our simulations are summarized in Tab. 2. We note that for both solid phases, we specify an upper and lower bound for the particle size PDF, to avoid generating non-realistic large/small particles. In our subsequent simulations, the initial packings are generated using the mass-in-flow (MIF) boundary condition (i.e., the particles are filled into the hopper from the top). In the current implementation, the MIF boundary condition does not allow one to precisely control the number of particles for each solid phase to be filled in the hopper. Therefore, the mass ratio is first determined in the hopper discharge simulation and then reproduced in the experiments, in which the mass for each phase can be controlled more precisely.

248 The simulations are carried out on Stampede at the Texas Advanced Computing Center (TACC), which consists of 64-bit Xeon E5-2680 2.7GHz (turbo, 3.5)

cores. The code is compiled with mvapich2/2.1.

Table 2: Geometrical parameters, masses of the two solid particle phases and global parameters used in validation simulations. 
Figure 7: Initial configuration of the discharge hopper containing a well-mixed packing of spherical beads with a bi-modal size distribution. The unit of the particle diameter is centimeter.

\subsection{Discharge hopper with polydisperse particles in well-mixed configuration}

hours for the discharge simulation are 6.73 hours.

Figure 8: Snapshots of the discharge simulation of the well-mixed configuration at $6 \mathrm{~s}, 9 \mathrm{~s}, 11$ $\mathrm{s}$ and $12 \mathrm{~s}$, respectively, from left to right. The upper panels show the top view and the lower panels show the side view.

Figure 9: Discharge dynamics are quantified using the normalized fine mass fraction $\gamma_{f}^{N}$ of the discharged particles vs. the overall discharged mass fraction $\bar{\gamma}$ for the well-mixed configuration. The experimental results are represented as a box plot.

Fig. 8 shows the snapshots of the discharge simulations at several different times. The discharge dynamics are quantified using the normalized fine mass 


\subsection{Discharge hopper with polydisperse particles in a layered configuration}

Figure 10: Initial configuration of the hopper discharge containing a layered packing of spherical beads with a bi-modal size distribution. The unit of the particle diameter is centimeter.

294

For the second validation case, we consider the discharge hopper with a layered configuration (see Fig. 10). The initial configuration for the discharge

fraction, $\gamma_{f}^{N}$, of the discharged particles vs. the overall discharged mass fraction, $\bar{\gamma}$, which are calculated by using Equations 1 and 2 .

uration stays well mixed, the resulting $\gamma_{f}^{N}-\bar{\gamma}$ curve would be a simple constant cluster at the hopper bottom towards the end of the discharge, leading to an increase of $\gamma_{f}^{N}$ towards the end of discharge (see Fig. 9p. As can been seen from the figure, our simulated $\gamma_{f}^{N}-\bar{\gamma}$ curve agrees very well quantitatively with our experimental data.

Nonetheless, it can be seen that the experimental data tends to deviate more from the ideally mixed case (i.e., the constant function), indicating a stronger segregation effect during the discharge. We note that in an "ideally mixed case", there will be no aggregation of particles of similar sizes during the entire discharge process. This situation is not possible in practice, as the particles during a discharge process always gain kinetic energy and thus, inevitably form aggregations of different sizes. The observed discrepancy is possibly because of the existence of locally segregated regions in the experimentally prepared initial packing configuration due to particle size dispersity, which is inevitable even though very careful mixing was conducted in the experiment. On the other hand, the particle mixing in the initial configuration can be much better controlled in the simulation through mass-in-flow boundary conditions. Therefore, the segregation in the simulated system is mainly due to discharge and becomes significant toward the end of the discharge process.

simulation is obtained by first filling the hopper with coarse beads from the top 
through the mass-in-flow boundary condition, while the bottom is kept closed

through the no-flow boundary condition, and then continuing to fill the hopper with fine beads, which pack on top of the coarse beads (see Fig. 10). The injection velocities of the fine and coarse beads through the top are $16 \mathrm{~cm} / \mathrm{s}$, with a volume fraction of 0.02 for each layer. The resulting packing is allowed to settle to completely dissipate the kinetic energy, and the resulting layered stable packing is used as the initial start for the discharge simulation until the hopper is fully discharged. The number of computational grids used in this simulation is 30 by 30 by 40 , along the two lateral directions and the axial direction of the hopper, respectively. The domain decomposition configuration is 4 by 4 by 4 with 64 cores and the total CPU hours for the discharge simulation are 6.81 hours.

Figure 11: Snapshots of the discharge simulation of the layered configuration at $6 \mathrm{~s}, 9 \mathrm{~s}, 11 \mathrm{~s}$ and $12 \mathrm{~s}$, respectively, from left to right. The upper panels show the top view and the lower panels show the side view.

Figure 12: Discharge dynamics are quantified using the normalized fine mass fraction $\gamma_{f}^{N}$ of the discharged particles vs. the overall discharged mass fraction $\bar{\gamma}$ for the layered configuration. The experimental results are represented as a box plot.

Fig. 11 shows the snapshots of the discharge simulations. The discharge dynamics as quantified via $\gamma_{f}^{N}-\bar{\gamma}$ are shown in Fig. 12. As seen in Fig. 11. in this case, the coarse particles packed at the hopper bottom discharge first, which opens up a channel for the fine particles on the top. Therefore, the resulting $\gamma_{f}^{N}$ remains zero for a while (before a channel is opened up), and is monotonically increasing as the discharge proceeds after the channel is open. Towards the end of discharge, since most of the fine particles in the hopper are gone, $\gamma_{f}^{N}$ begins to decrease and finally starts to fluctuate due to a very small number of fine particles remaining in the hopper. Again, our simulated $\gamma_{f}^{N}-\bar{\gamma}$ curve agrees very well quantitatively with the experimental data, as shown in Fig. 12.

Our simulation also reveals how the channel for fine particles is opened up 
during the discharge. Due to the geometry of the hopper, the particles in the central region corresponding to a virtual extension of the cylinder-shaped outlet at the hopper bottom always discharge first, regardless of the initial packing configurations in the hopper. The fast flow of discharging particles in this central region generates a pressure on the remaining particles and pushes them against the container wall. In the layered configuration, the coarse particles are packed at the bottom of the hopper and fine particles are then stacked on top. Thus, once the discharge process starts, the coarse particles in the central region discharge first, which efficiently open up a channel for the fine particles that were originally packed on top of the coarse ones.

Again, it can be seen that the experimental data tends to deviate more from the ideally mixed case (i.e., unity) compared to the simulation results. Although, in this layered configuration, the coarse and fine particles are separately packed in their own layers, within each layer the locally segregated regions still exist (due to the polydispersity of particles within the same solid phase) in the experimentally prepared initial configurations. This leads to the observed stronger segregation effects during the discharge process. For better controlled initial configurations in the simulations, the segregation is better suppressed and thus, the deviation of the simulated results from the ideally mixed case is less significant.

\section{Summary and Concluding Remarks}

In this paper, we presented our recent developments for enhancing MFIXDEM's capability for handling particle size polydispersity for the pure DEM mode flow simulations. We have modified the data structure and created new subroutines to separately handle geometrical and physical parameters of the solid phase particles. This allows MFIX-DEM to easily handle an arbitrary number of solid phases, each possessing a distinct arbitrary particle-size distribution. New keywords have been introduced to allow easy specification of a variety of built-in and user-defined distributions, for both the initial condition 
and mass-in-flow boundary condition. The new implementation is systemati-

cally validated using discharge hopper experiments, a commonly encountered set-up with many industrial applications, with polydisperse particles. The simulated discharge dynamics, including the normalized fine-mass-fraction vs. the overall discharged mass fraction, are found to be in reasonably good agreement with the corresponding experimental data, for the purposes of the preliminary V\&V study. To better understand discrepancy observed and assess the potential sources, based on the insight gained from the current work, a follow-up uncertainty quantification study is being employed by utilizing global sensitivity analysis to determine which set of factors have the most influence on the normalized fine-mass-fraction vs. the overall discharged mass fraction ratio. For this purpose, an ensemble simulation matrix based on statistical design of experiments is being carried out to explore the parameter space (e.g., spring coefficient variables range which is used in contact models) and assess the sensitivities of the quantity of interest. The findings of the sensitivity study will pave the way for our future efforts on reducing the uncertainty in modeling parameters by employing techniques like Bayesian calibration.

The current implementation can easily handle particle size dispersity as large as 10:1. To efficiently handle larger dispersity (e.g., $>20: 1$ ), additional modifications of the codes (e.g., by implementing adaptive near-neighbor list [39]) are required. Nonetheless, the new polydispersity feature added to MFIX-DEM enables users to better capture the actual particle geometry of the solid phases, and thus, would lead to more accurate simulations of multiphase flows involving particle phases, especially in the dense flow regime. The current implementation will also pave the way for the non-spherical and reacting flow related revisions, as the data structures for handling individual particle sizes can be readily generalized to handle other particle geometry parameters and chemical reaction related parameters. This will allow the users to specify individual particle-based physical properties. We will explore these additional enhancements to the physical modeling capability of MFIX-DEM in our future work. 


\section{Acknowledgment}

380 François Dietiker of WVURC/NETL and Dr. Tingwen Li of AECOM/NETL

The authors are very grateful to Dr. Jordan Musser of NETL, Dr. Jeanfor their kind help and valuable discussions. This research effort is funded by the U.S. Department of Energy's National Energy Technology Laboratory (NETL) Crosscutting Research Program Transitional Technology Development to Enable Highly Efficient Power Systems with Carbon Management initiative under award DE-FE0026393. This research used resources of the National Energy Research Scientific Computing Center (NERSC), a DOE Office of Science User Facility supported by the Office of Science of the U.S. Department of Energy under Contract No. DE-AC02-05CH11231. This work has also used the Extreme Science and Engineering Discovery Environment (XSEDE), which is supported by National Science Foundation grant number ACI-1053575.

\section{References}

[1] M. Jahani, A. Farzanegan, M. Noaparast, Investigation of screening performance of banana screens using liggghts dem solver, Powder Technology 283 (2015) 32-47.

[2] Y. Gu, A. Ozel, S. Sundaresan, A modified cohesion model for cfd-dem simulations of fluidization, Powder Technology 296 (2016) 17-28.

[3] J. C. Loh, W. R. Ketterhagen, J. A. Elliott, A hybrid tabulation-scaling implementation of thornton and ning's plastic-adhesive particle contact theory, Powder Technology 264 (2014) 599-607.

[4] P. L. Falkingham, S. M. Gatesy, The birth of a dinosaur footprint: Subsurface $3 \mathrm{~d}$ motion reconstruction and discrete element simulation reveal track ontogeny, Proceedings of the National Academy of Sciences 111 (51) (2014) 18279-18284. 
[5] T. Kopsch, D. Murnane, D. Symons, Optimizing the entrainment geometry

[10] M. Syamlal, W. Rogers, T. J. O'Brien, MFIX documentation theory guide of a dry powder inhaler: Methodology and preliminary results, Pharmaceutical Research (2016) 1-12.

[6] H. S. Rabbani, V. Joekar-Niasar, N. Shokri, Effects of intermediate wettability on entry capillary pressure in angular pores, Journal of colloid and interface science 473 (2016) 34-43.

[7] A. Sharifi, H. Niazmand, Analysis of flow and ldl concentration polarization in siphon of internal carotid artery: Non-newtonian effects, Computers in biology and medicine 65 (2015) 93-102.

[8] MFIX-GUI, url = https://mfix.netl.doe.gov/mfix/mfix-gui-2/.

[9] R. Garg, J. Galvin, T. Li, S. Pannala, Documentation of open-source MFIX-DEM software for gas-solids flows.

URL https://mfix.netl.doe.gov/documentation/dem_doc_2012-1. pdf URL https://mfix.netl.doe.gov/documentation/Theory.pdf

[11] T. Li, W. A. Rogers, M. Syamlal, J.-F. Dietiker, J. Musser, M. Shahnam, S. Rabha, The NETL MFIX Suite Of Multiphase Flow Models: A Brief Review And Recent Applications Of MFIX-TFM To Fossil Energy Technologies, Chemical Engineering Sciencedoi:10.1016/j.ces.2016.07.043

[12] W. Rogers, NETL Multiphase Flow Research Overview, in: Presented as part of the NETL Workshop on Multiphase Flow Science, National Energy Technology Laboratory, 2016.

[13] M. Syamlal, C. Guenther, A. Gel, S. Pannala, Advanced coal gasifier designs using large-scale simulations, in: Journal of Physics: Conference Series, Vol. 180, IOP Publishing, 2009, p. 012034. 
[14] A. Gel, R. Sankaran, S. Pannala, C. Guenther, Accelerating clean coal gasifier designs with hybrid MPI/OpenMP high performance computing, Parallel Computational Fluid Dynamics: Recent Advances and Future Directions (2010) 372.

[15] E. F. D'Azevedo, S. Pannala, M. Syamlal, A. Gel, M. Prinkey, T. J. O'Brien, Parallelization of MFIX: A multiphase cfd code for modeling fluidized beds, in: Tenth SIAM Conference on Parallel Processing for Scientific Community (PPSC), 2001.

[16] M. Syamlal, T. J. O'Brien, Fluid dynamic simulation of $o_{3}$ decomposition in a bubbling fluidized bed, AIChE Journal 49 (2003) 2793-2801.

[17] T. Li, C. Guenther, MFIX-DEM simulations of change of volumetric flow in fluidized beds due to chemical reactions, Powder Technology 220 (2012) 70-78.

[18] R. Garg, J. Galvin, T. Li, S. Pannala, Open-source MFIX-DEM software for gas-solids flows: Part i. verification studies, Powder Technology 220 (2012) 122-137.

[19] T. Li, R. Garg, J. Galvin, S. Pannala, Open-source MFIX-DEM software for gas-solids flows: Part ii. validation studies, Powder Technology 220 (2012) 138-150.

[20] P. Liu, C. M. Hrenya, Challenges of DEM: I. competing bottlenecks in parallelization of gas-solid flows, Powder Technology 264 (2014) 620-626.

[21] J. Musser, Modeling of heat transfer and reactive chemistry for particles in gas-solid flow utilizing continuum-discrete methodology (cdm), Ph.D. thesis, West Virginia University Libraries (2011).

[22] A. Gel, J. Hu, Scaling an open source cfd code by leveraging parallel open source solvers within the Trilinos framework-preliminary results, in: Proceedings of the 2011 Parallel CFD Conference, Barcelona, Spain, 2011. 
[23] A. Gel, E. Ould-Ahmed-Vall, A. Kalinkin, Optimization Of A Legacy Open Source CFD Code For The New High Performance Computing Architectures: Preliminary Results, 27th International Conference on Parallel Computational Fluid Dynamics Conference (ParallelCFD 2015), Montreal, Canada, 2015.

[24] A. Gel, J. Hu, Modernizing A Legacy Open Source CFD Code By Leveraging Scalable Parallel Preconditioners And Linear Equation Solvers, 27th International Conference on Parallel Computational Fluid Dynamics Conference (ParallelCFD 2015), Montreal, Canada, 2015.

[25] A. Gel, R. Sankaran, S. Pannala, C. Guenther, Accelerating clean coal gasifier designs with hybrid mpi/openmp high performance computing, Parallel Computational Fluid Dynamics: Recent Advances and Future Directions (2010) 372.

[26] S. Pannala, E. D’Azevedo, M. Syamlal, Hybrid (OpenMP and MPI) parallelization of MFIX: a multiphase cfd code for modeling fluidized beds, in: Proceedings of the 2003 ACM symposium on Applied computing, ACM, 2003, pp. 199-206.

[27] H. Liu, D. K. Tafti, T. Li, Hybrid parallelism in MFIX cfd-dem using openmp, Powder Technology 259 (2014) 22-29.

[28] P. Gopalakrishnan, D. Tafti, Development of parallel dem for the open source code MFIX, Powder Technology 235 (2013) 33-41.

[29] P. Gopalakrishnan, D. Tafti, Development of parallel dem for the open source code MFIX, Powder Technology 235 (2013) 33-41.

[30] H. Liu, D. Tafti, T. Li, Hybrid parallelism in MFIX CFD-DEM using OpenMP, Powder Technology 259 (2014) 22-29.

[31] A. Gel, M. Shahnam, J. Musser, A. K. Subramaniyan, J.-F. Dietiker, Nonintrusive uncertainty quantification of computational fluid dynamics sim- 
ulations of a bench-scale fluidized-bed gasifier, Industrial \& Engineering

Chemistry Research 55 (2016) 12477-12490.

[32] A. Gel, R. Garg, C. Tong, M. Shahnam, C. Guenther, Applying uncertainty quantification to multiphase flow computational fluid dynamics, Powder Technology 242 (2013) 27-39.

[33] D. C. Miller, B. Ng, J. Eslick, Advanced computational tools for optimization and uncertainty quantification of carbon capture processes, Tech. rep., National Energy Technology Laboratory-In-house Research; National Energy Technology Laboratory (NETL), Pittsburgh, PA, and Morgantown, WV (United States) (2014).

[34] J. C. Eslick, B. Ng, Q. Gao, C. H. Tong, N. V. Sahinidis, D. C. Miller, A framework for optimization and quantification of uncertainty and sensitivity for developing carbon capture systems, Energy Procedia 63 (2014) 1055-1063.

[35] K. J. Berger, C. M. Hrenya, Challenges of dem: Ii. wide particle size distributions, Powder Technology 264 (2014) 627-633.

[36] Y. Jiao, F. H. Stillinger, S. Torquato, Distinctive features arising in maximally random jammed packings of superballs, Physical Review E 81 (2010) 041304.

[37] C. Kloss, C. Goniva, A. Hager, S. Amberger, S. Pirker, Models, algorithms and validation for opensource dem and cfd-dem, Progress in Computational Fluid Dynamics, an International Journal 12 (2-3) (2012) 140-152.

[38] H. N. Emady, K. V. Anderson, W. G. Borghard, F. J. Muzzio, B. J. Glasser, A. Cuitino, Prediction of conductive heating time scales of particles in a rotary drum, Chemical Engineering Science 152 (2016) 45-54.

[39] A. Donev, S. Torquato, F. H. Stillinger, Neighbor list collision-driven molecular dynamics for nonspherical hard particles: Ii. applications to ellipses and ellipsoids, Journal of Computational Physics 202 (2005) 765. 
[40] Y. Jiao, F. H. Stillinger, S. Torquato, Dense packings of superballs, Physical

[41] Y. Jiao, S. Torquato, Dense packings of platonic and archimedean solids,

[42] P. Von Zedtwitz, W. Lipiński, A. Steinfeld, Numerical and experimental study of gas-particle radiative heat exchange in a fluidized-bed reactor for steam-gasification of coal, Chemical engineering science 62 (1) (2007) 599607.

[43] J. Dufek, G. Bergantz, Transient two-dimensional dynamics in the upper conduit of a rhyolitic eruption: A comparison of closure models for the granular stress, Journal of Volcanology and Geothermal Research 143 (1) (2005) 113-132.

[44] M. R. Sweeney, G. A. Valentine, Transport and mixing dynamics from explosions in debris-filled volcanic conduits: numerical results and implications for maar-diatreme volcanoes, Earth and Planetary Science Letters 425 (2015) 64-76.

[45] J. Estep, J. Dufek, Discrete element simulations of bed force anomalies due to force chains in dense granular flows, Journal of Volcanology and Geothermal Research 254 (2013) 108-117.

[46] S. Dartevelle, From model conception to verification and validation, a global approach to multiphase navier-stoke models with an emphasis on volcanic explosive phenomenology, Tech. rep., Los Alamos National Laboratory (LANL), Los Alamos, NM (2007).

[47] S. Dartevelle, G. Valentine, Transient multiphase processes during the explosive eruption of basalt through a geothermal borehole (námafjall, iceland, 1977) and implications for natural volcanic flows, Earth and Planetary Science Letters 262 (3) (2007) 363-384. 
540

542

544

546

548

[48] M. D. Team, Multiphase Flow with Interphase eXchanges (MFIX) user guide, National Energy Technology Laboratory, Morgantown, WV.

[49] J. F. Dietiker, Multiphase flow with interphase exchanges cartesian grid user guide, National Energy Technology Laboratory, Morgantown, WV.

[50] W. R. Ketterhagen, J. S. Curtis, C. R. Wassgren, A. Kong, P. J. Narayan, B. C. Hancock, Chemical Engineering Science.

[51] N. Standish, A. Kilic, Comparison of stop, start and continuous sampling methods of studying segregation of materials discharging from a hopper, Chemical engineering science 40 (11) (1985) 2152-2153. 


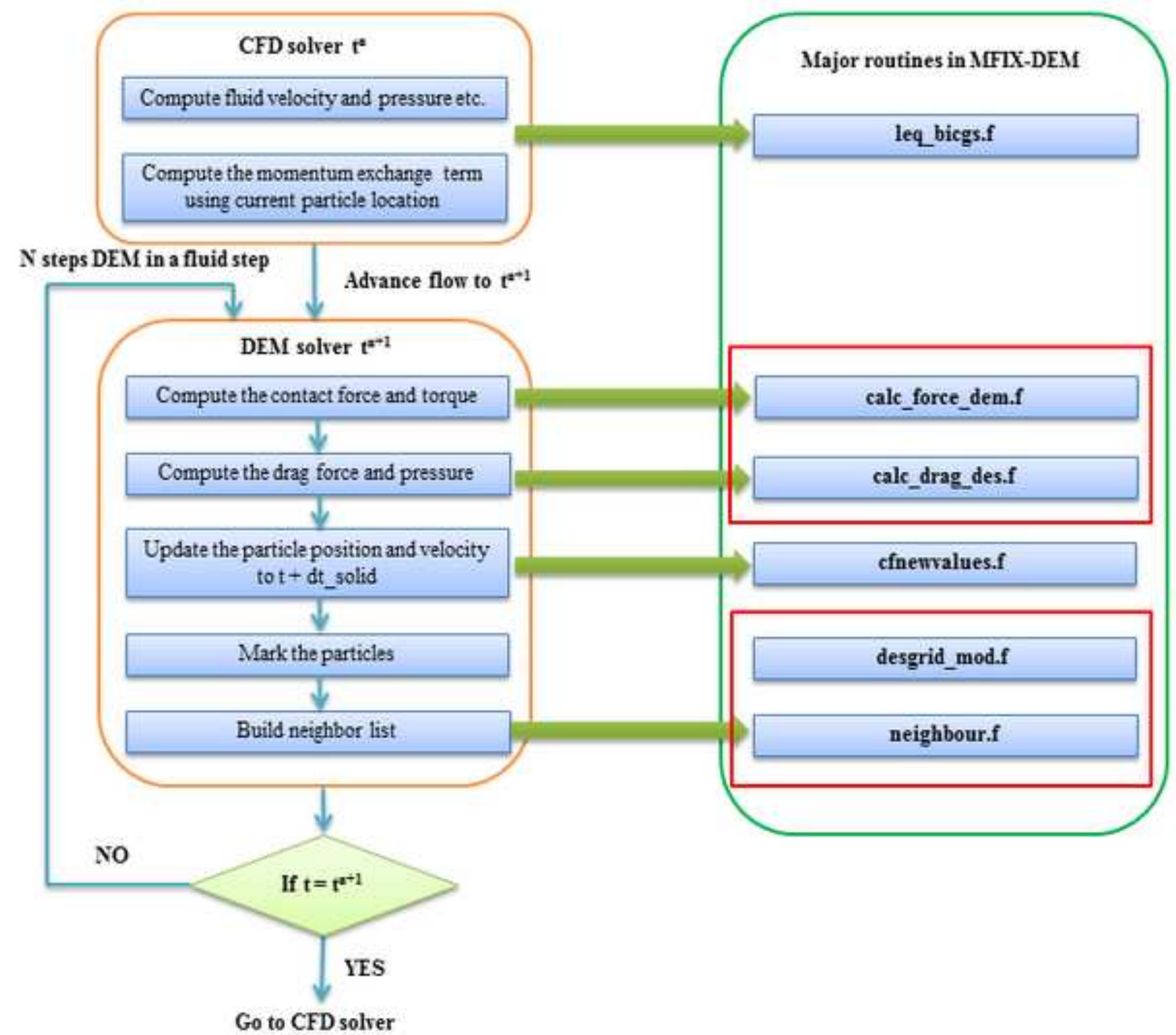




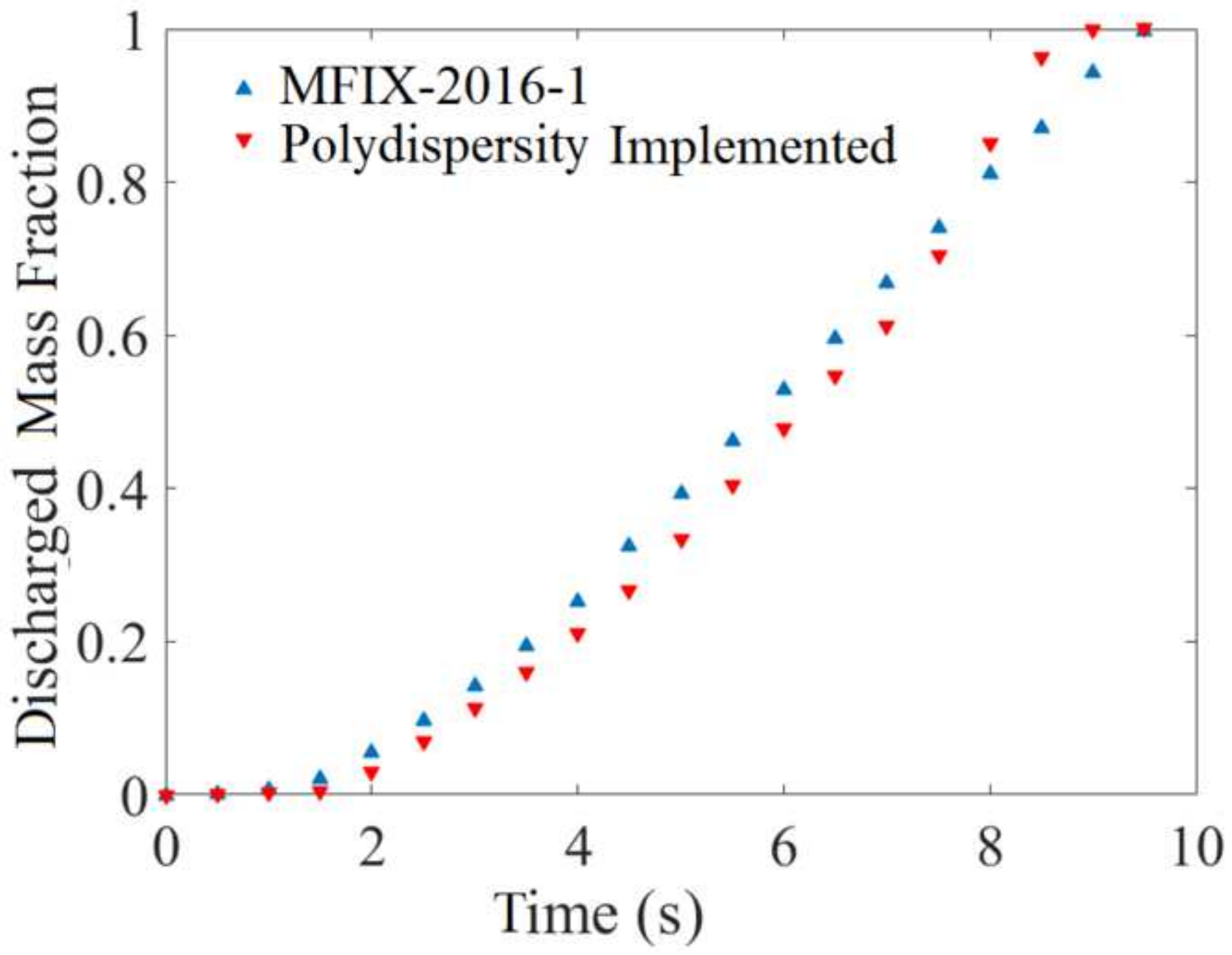




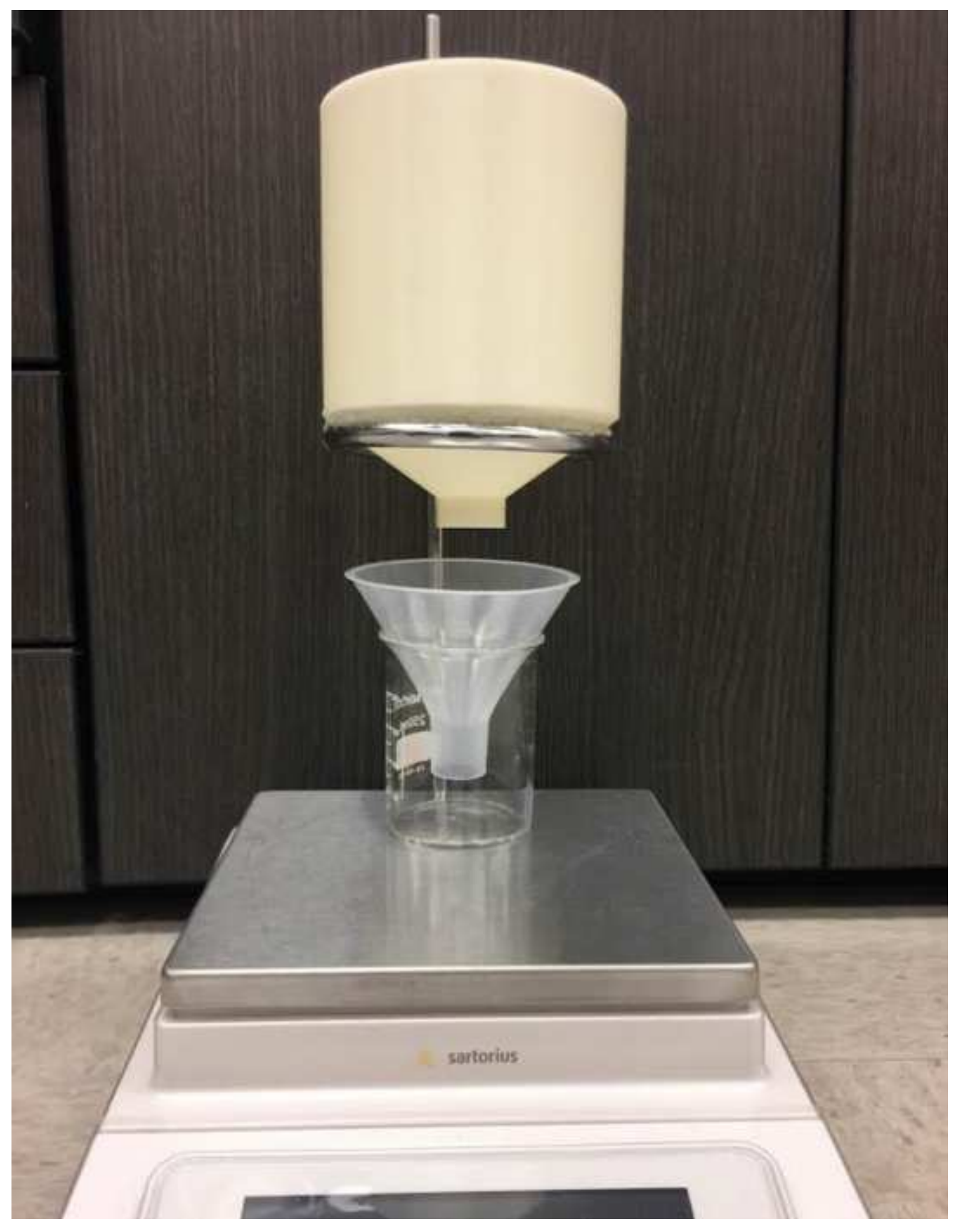




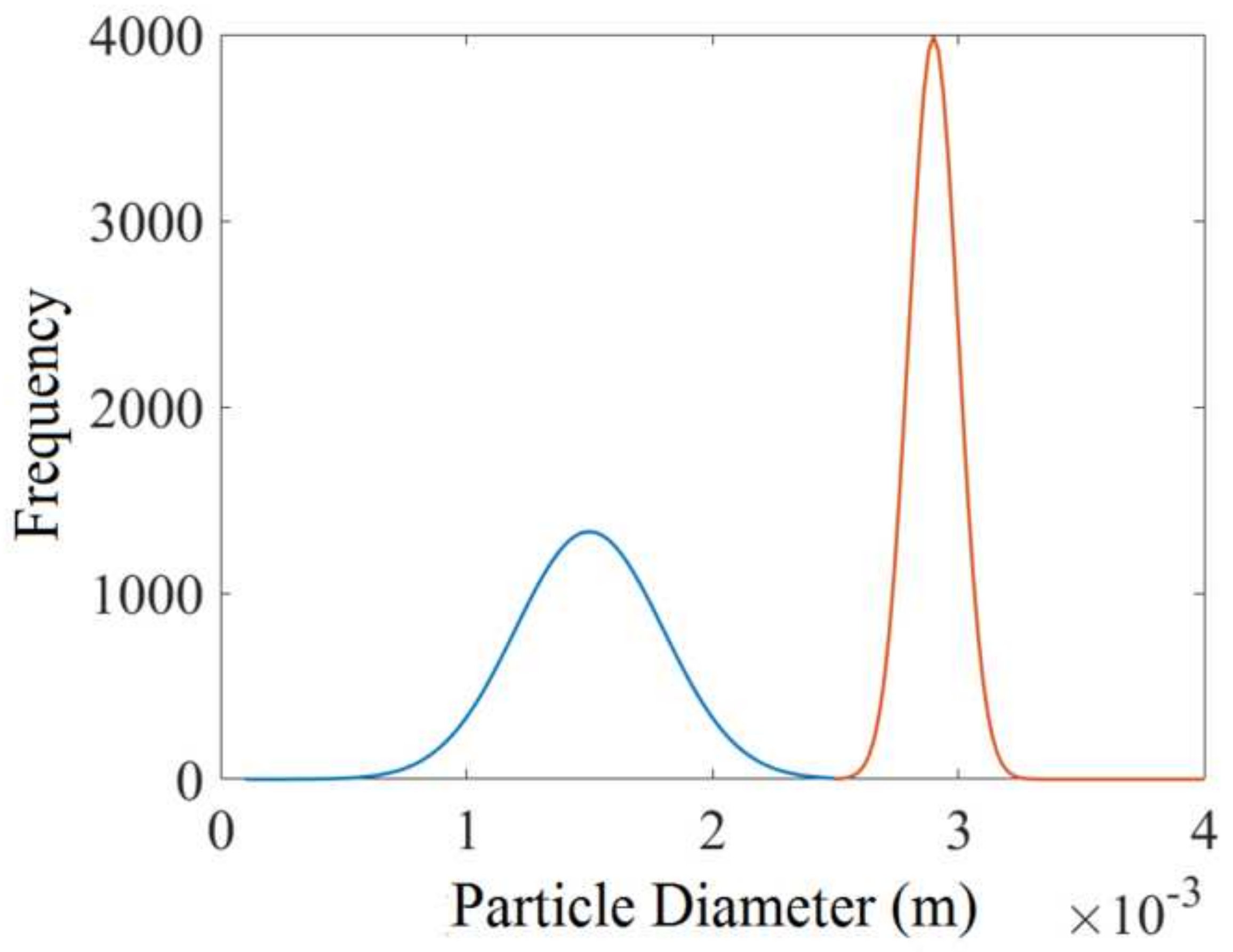




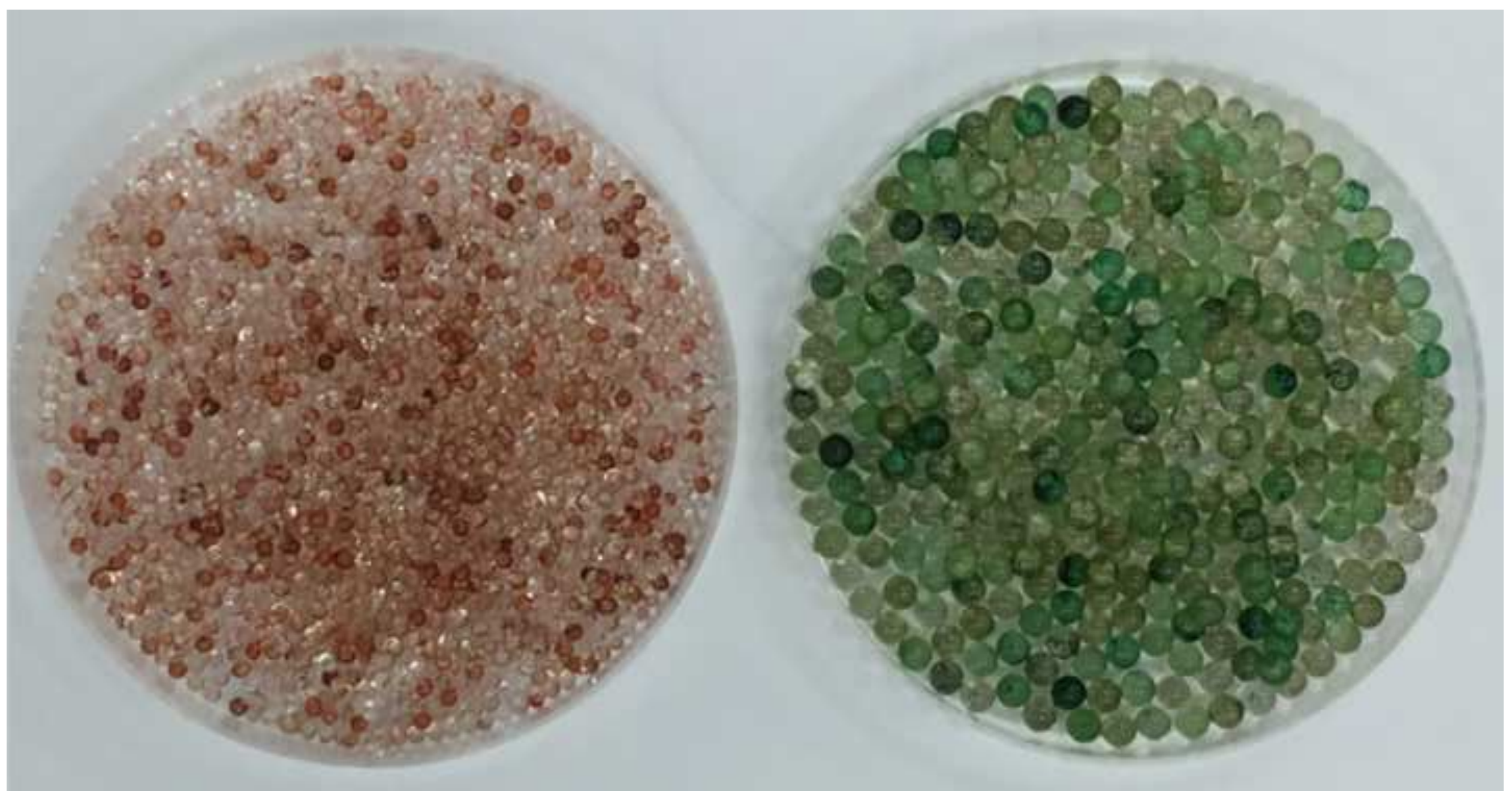




\section{Well-Mixed Configuration}
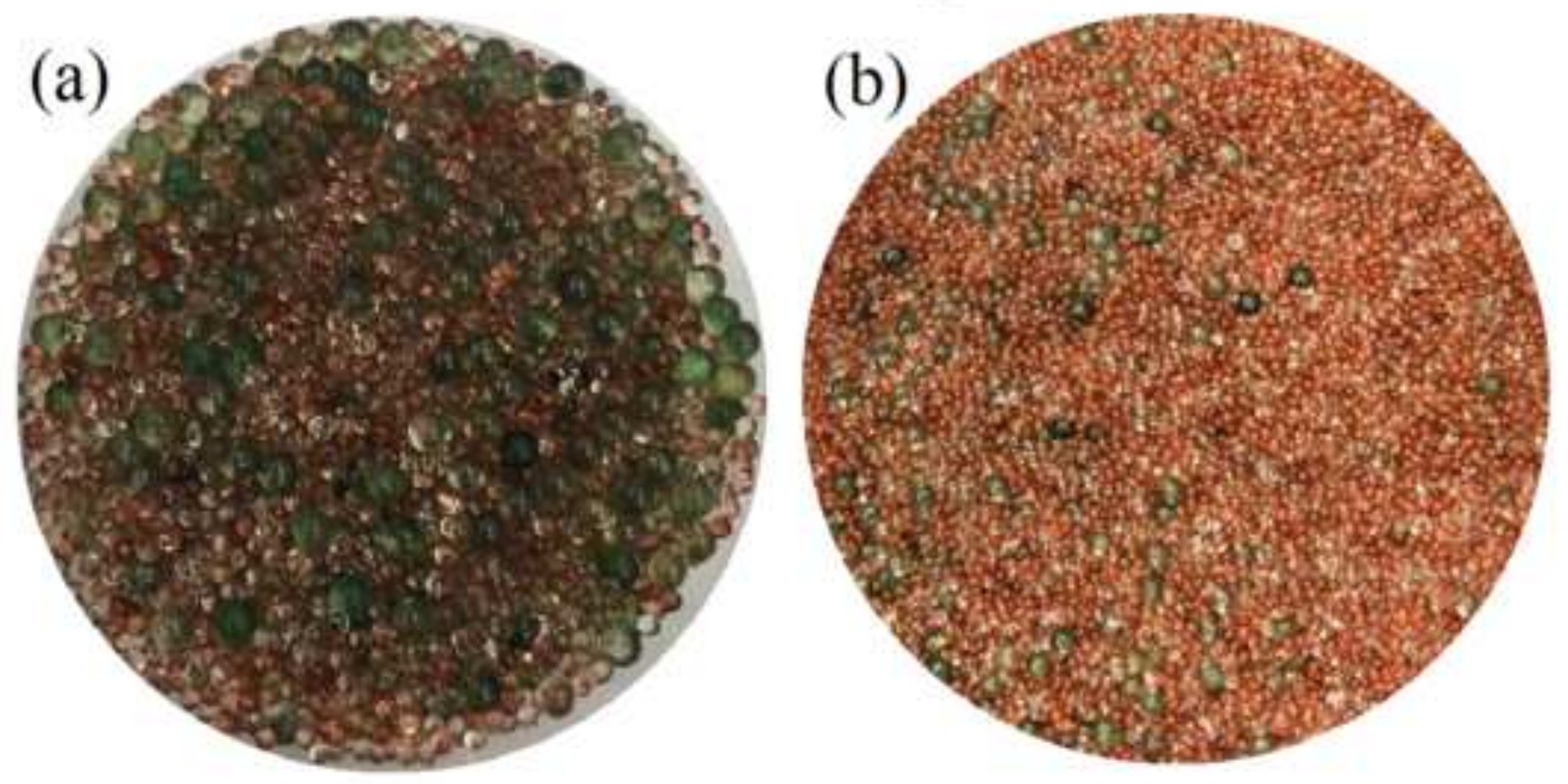

\section{Layered Configuration}
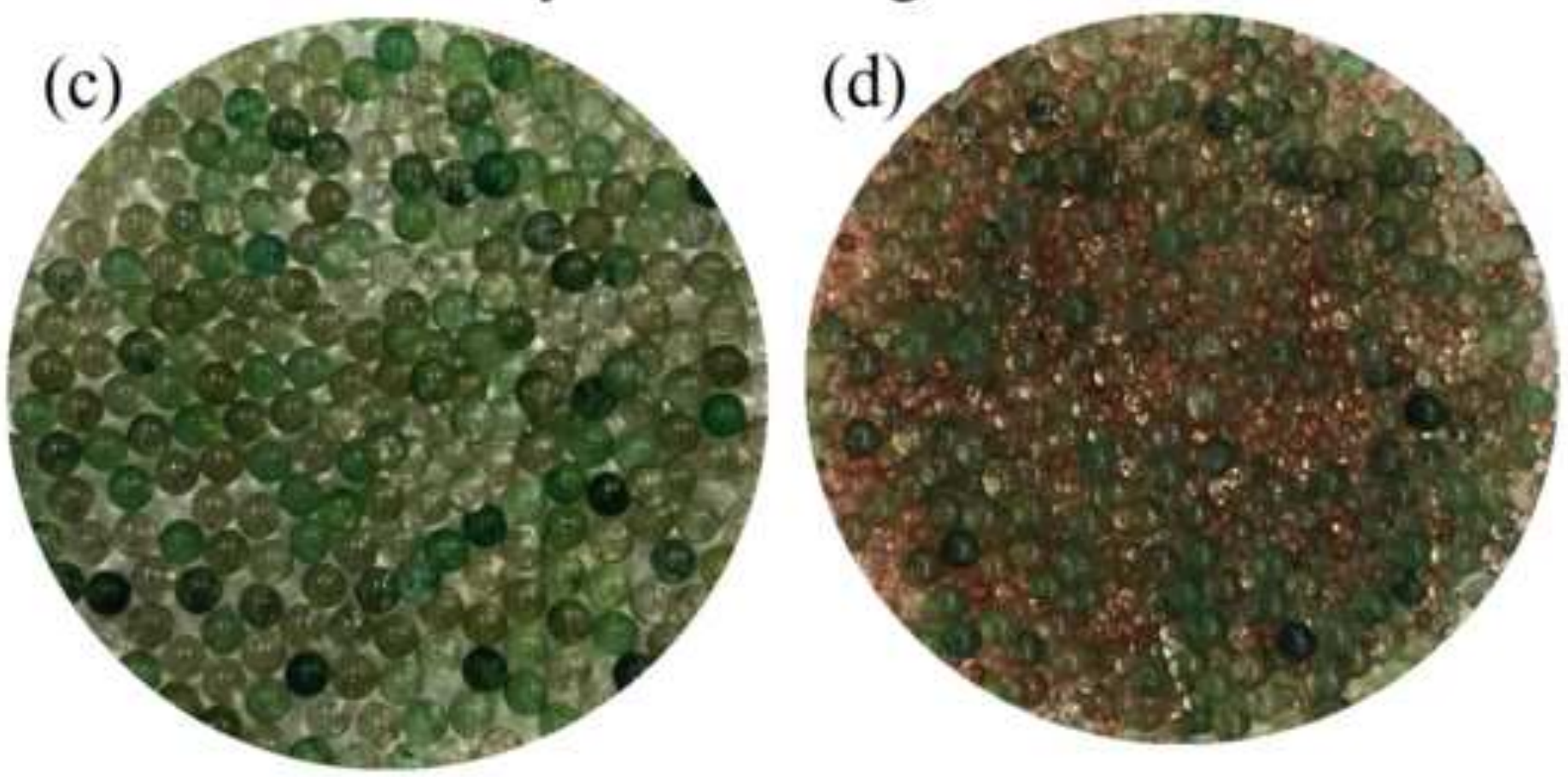


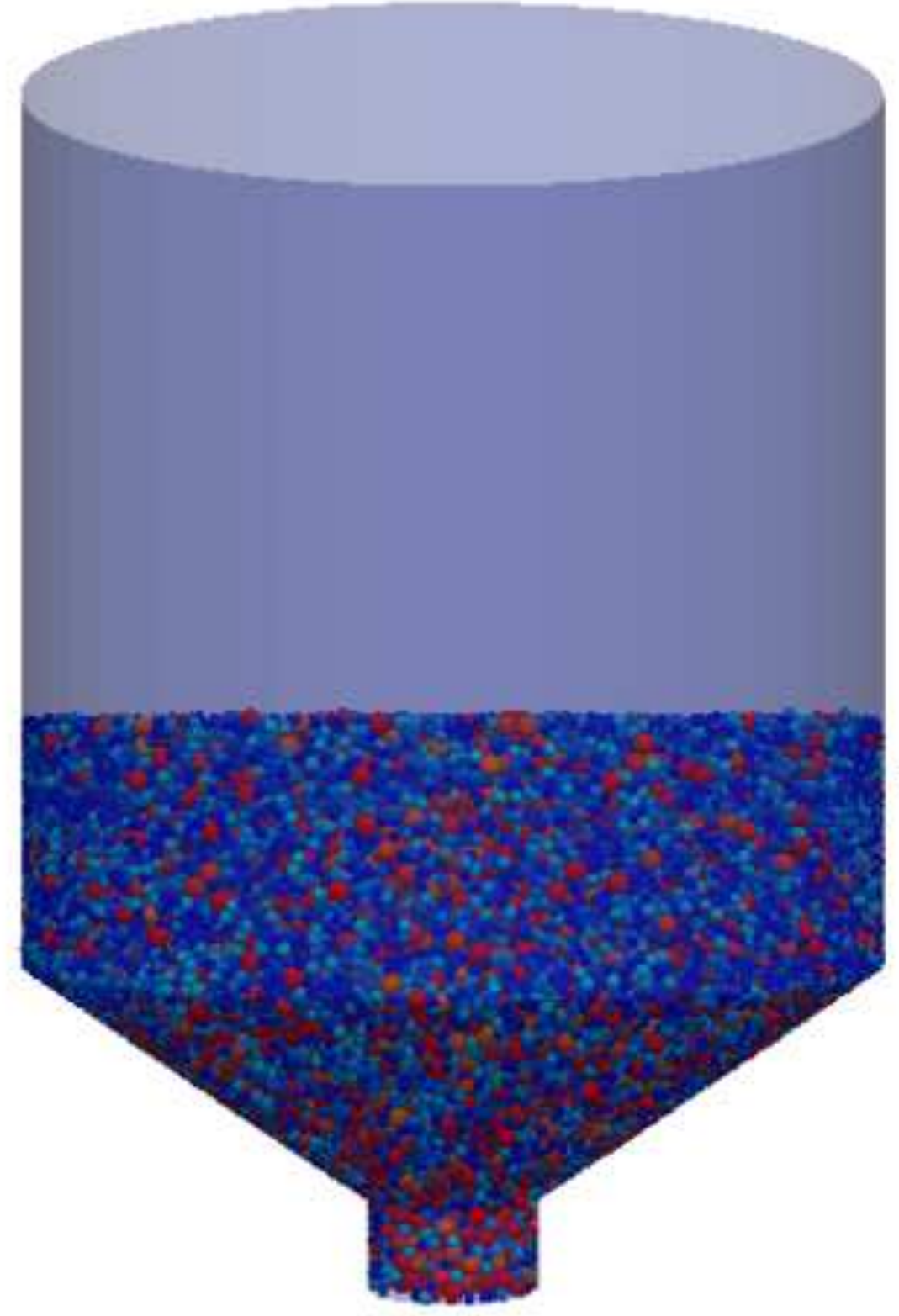

Diameter (cm)

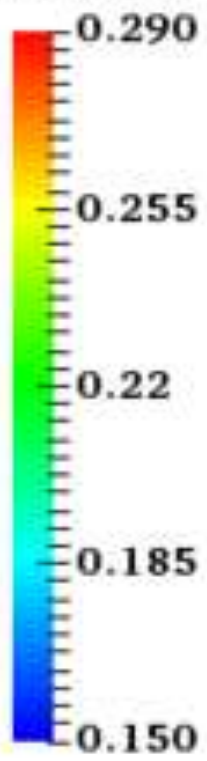



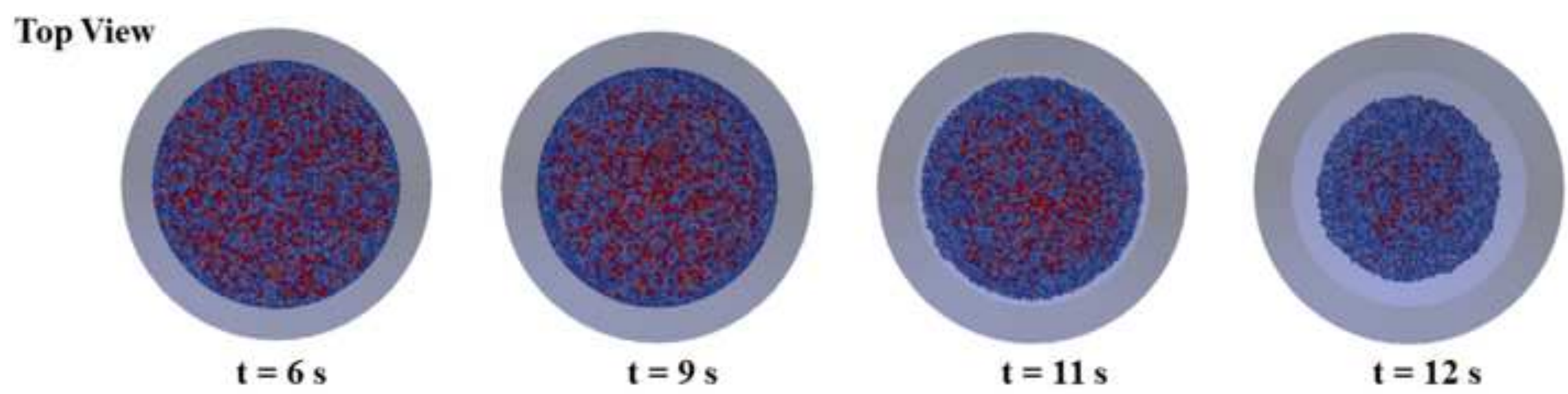

\section{Front View}
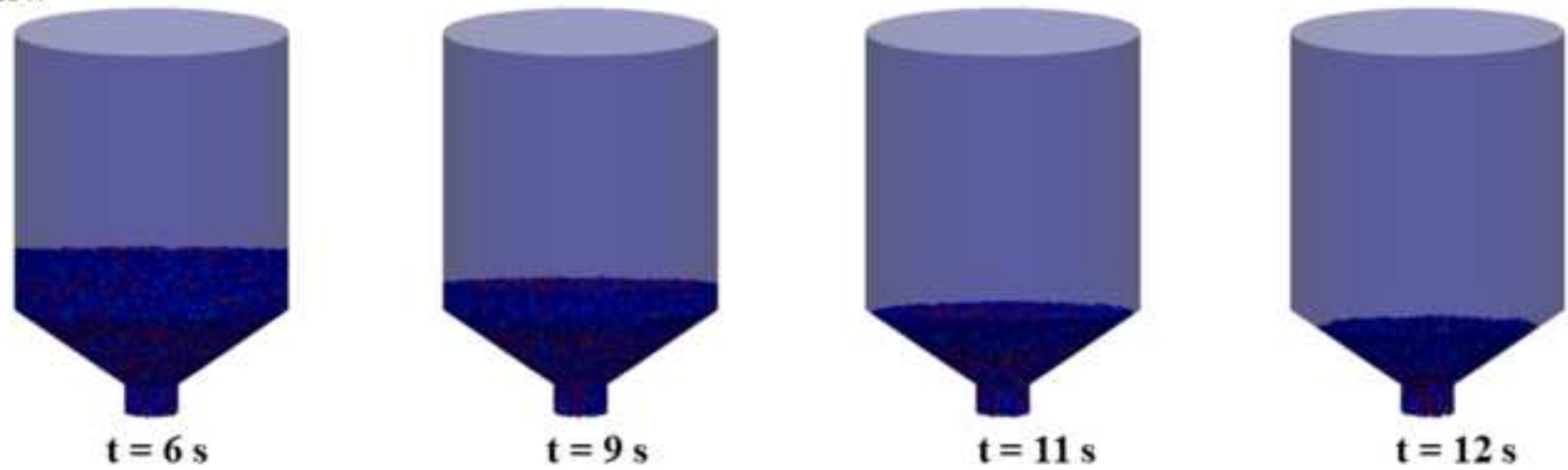


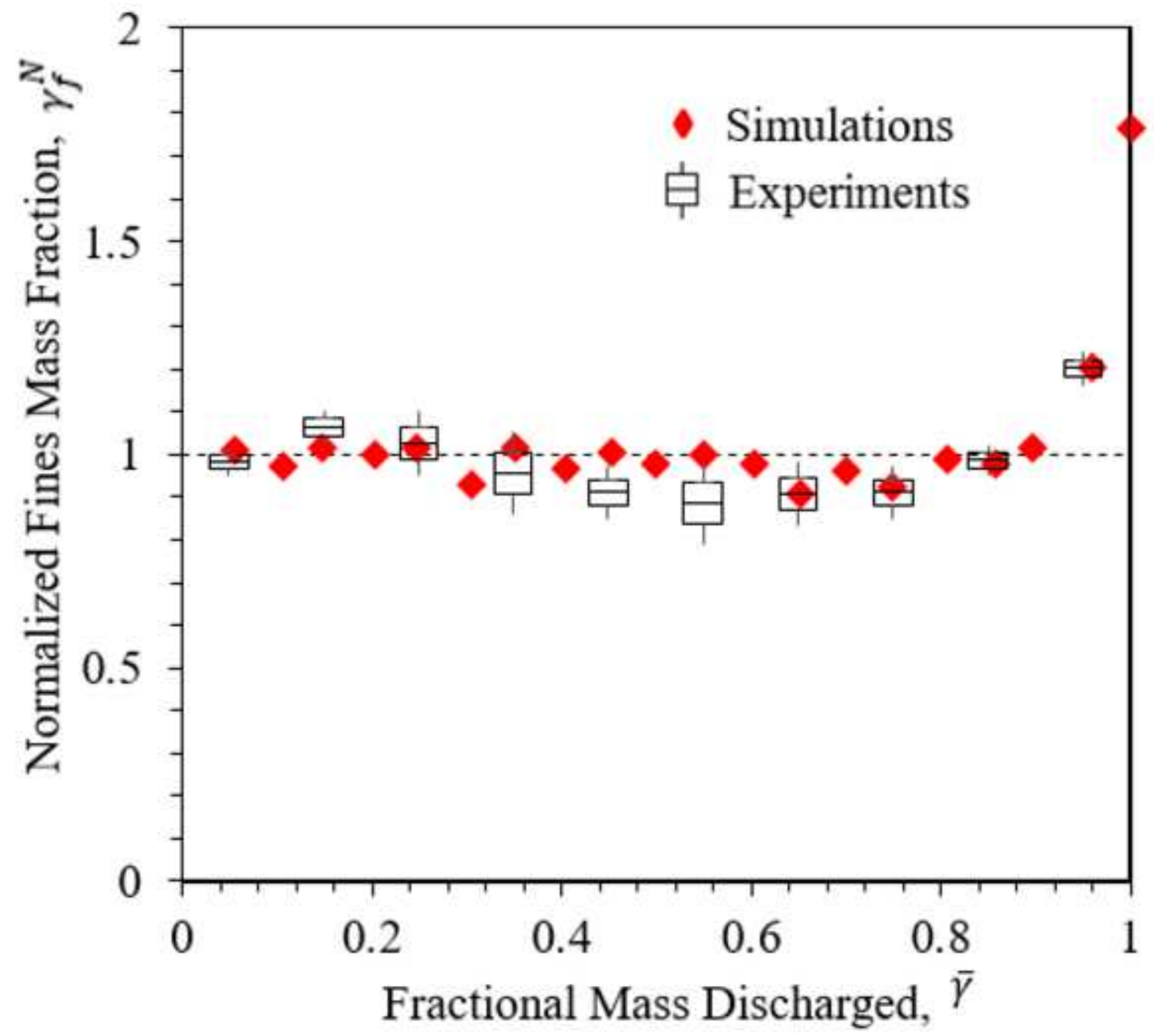




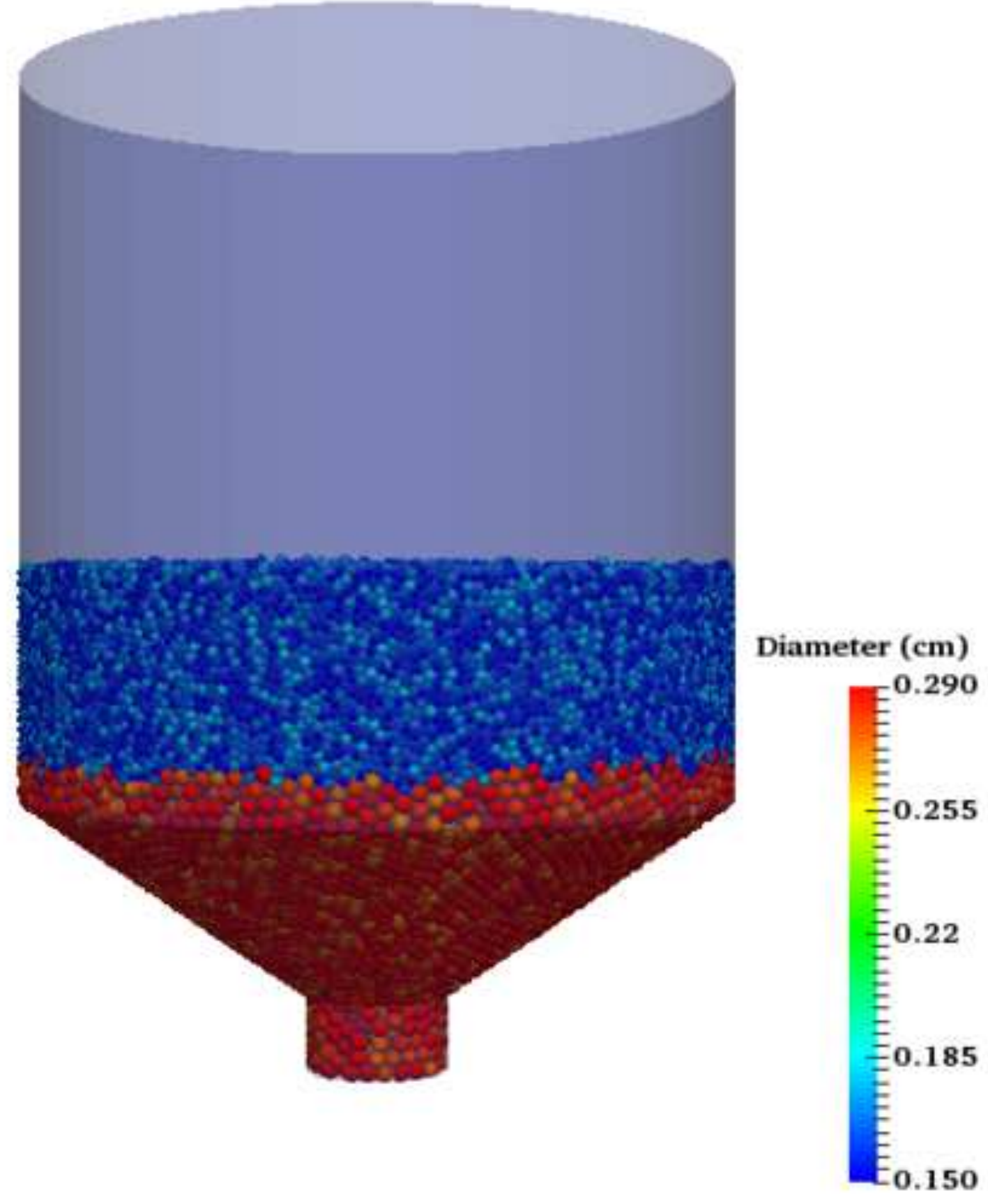



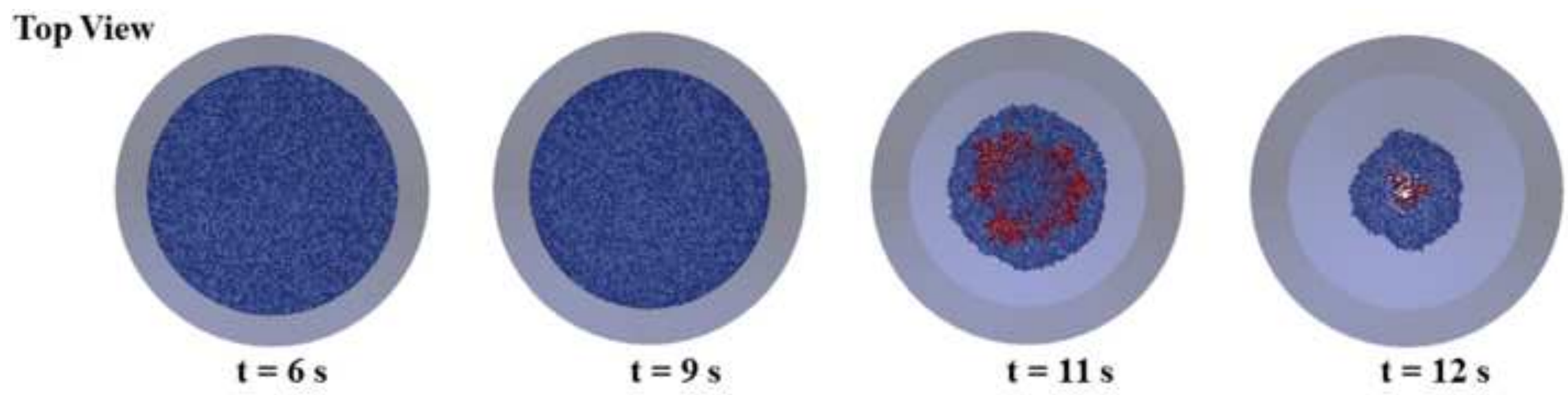

\section{Front View}
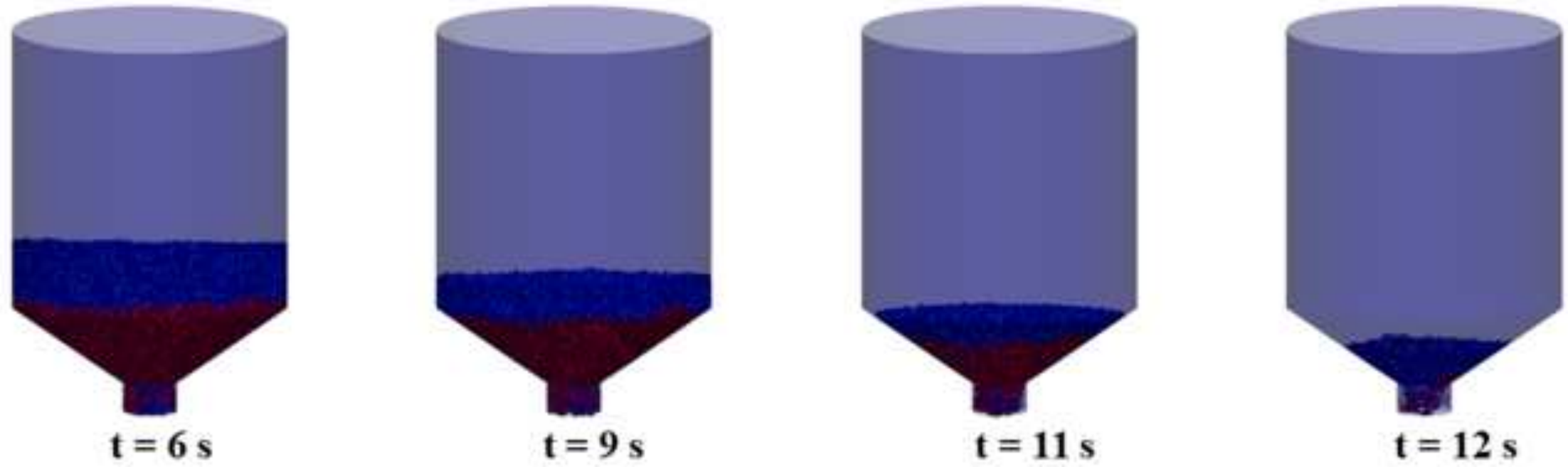


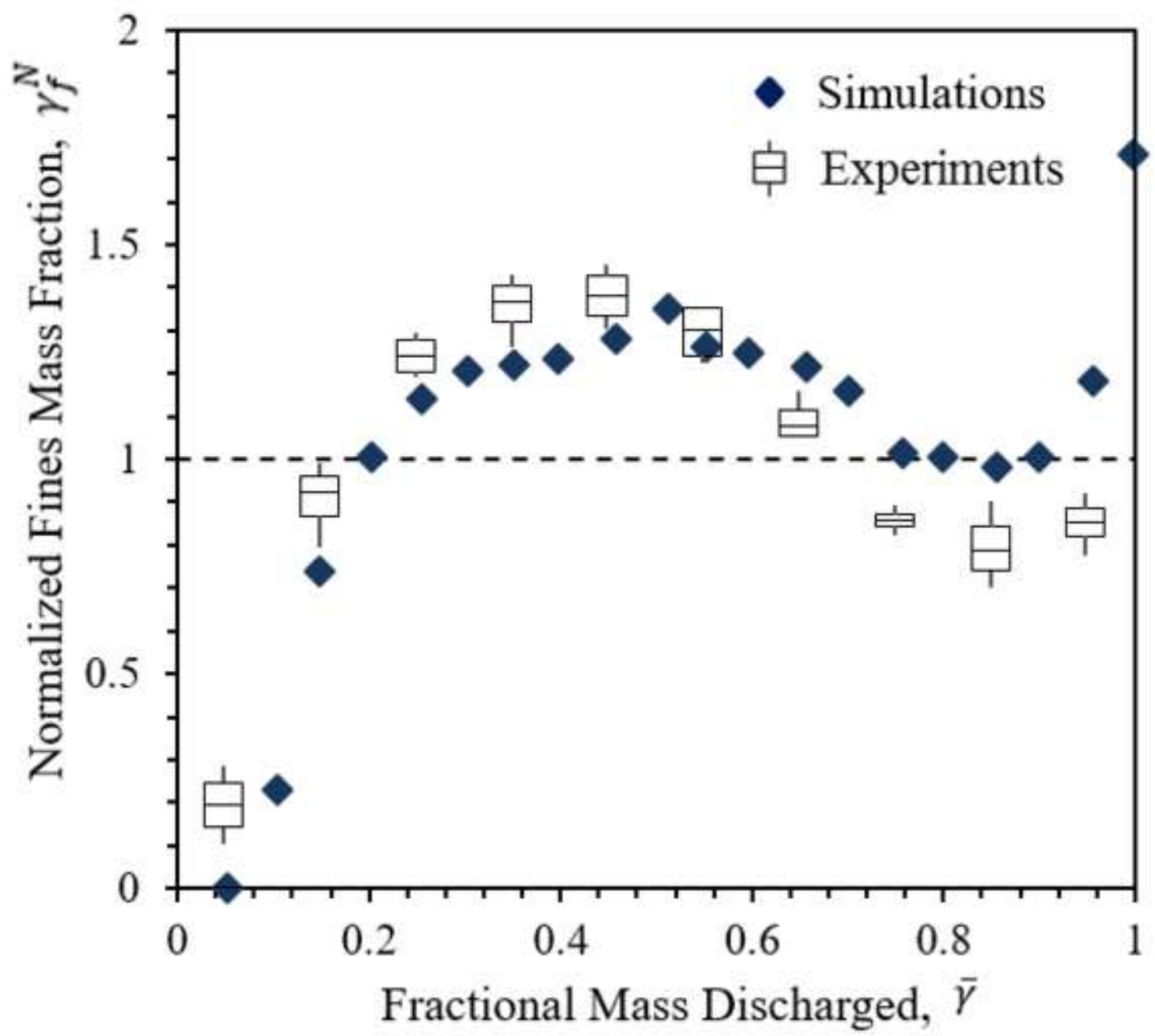


Domain Decomposition Total Number CPU

Configuration of Particles hours

\begin{tabular}{llll}
\hline MFIX-DEM 2016-1 & $2 \times 2 \times 2$ & 15544 & 5.45 \\
Our implementation & $2 \times 2 \times 2$ & 15540 & 5.44 \\
\hline \hline
\end{tabular}




\section{Sample}

Mean Dia.

Max Dia.

Min Dia.

Std. Dev.

Total Mass

Spring Constant

Friction Coefficient

Coefficient of Restitution
Fine

Coarse

$0.15 \mathrm{~cm}$

$0.29 \mathrm{~cm}$

$0.17 \mathrm{~cm}$

$0.31 \mathrm{~cm}$

$0.13 \mathrm{~cm}$

$0.27 \mathrm{~cm}$

$0.03 \mathrm{~cm}$

$0.01 \mathrm{~cm}$

$580 \mathrm{~g}$

$420 \mathrm{~g}$

$2.5 \times 10^{5} \mathrm{~g} / \mathrm{s}^{2}$

$2.5 \times 10^{5} \mathrm{~g} / \mathrm{s}^{2}$

0.5

0.5

0.9

0.9 

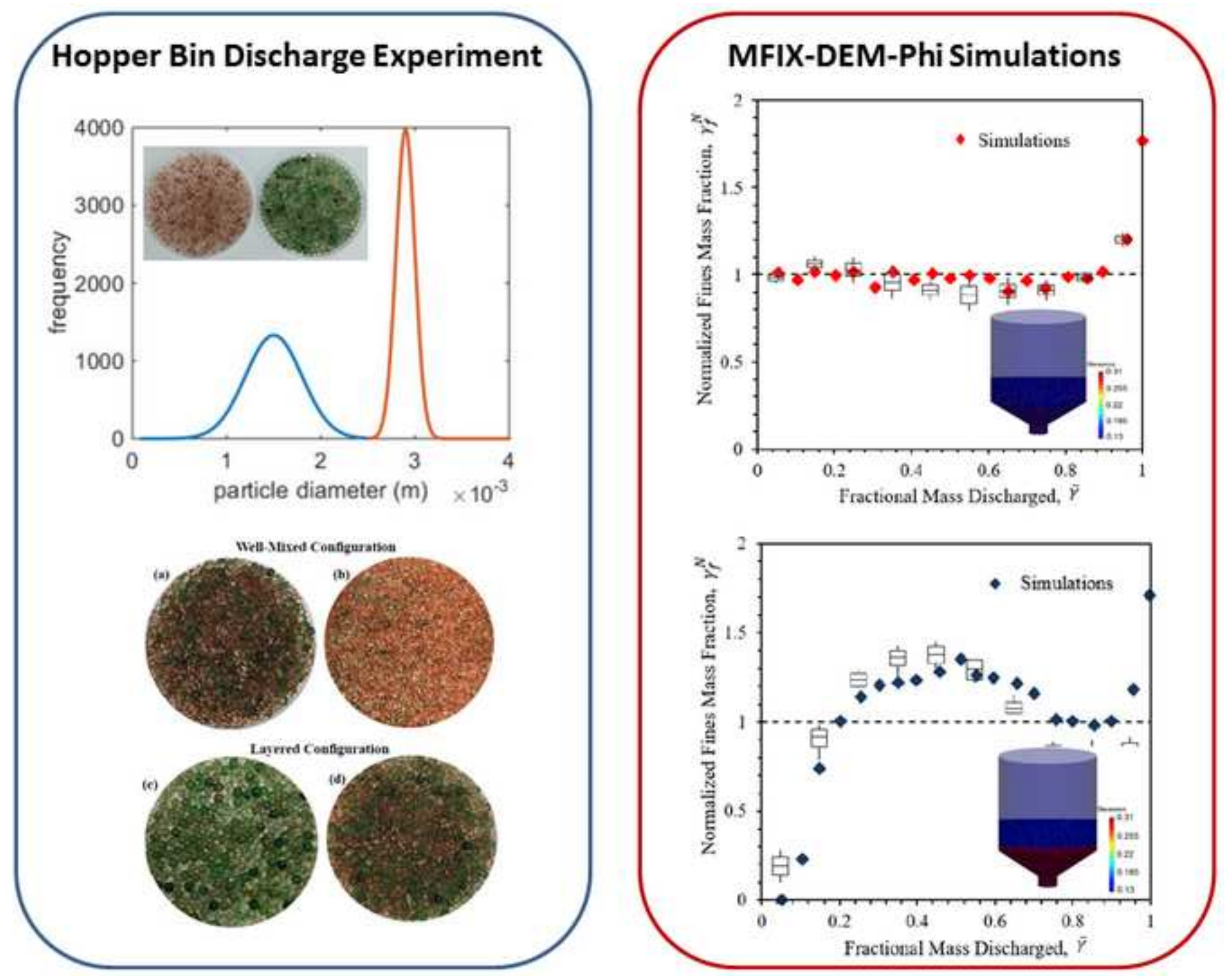